\title{
Translatability Problem of Geodesics on Algorithmic Manifolds
}

\author{
Takuya Yabu(takuya.yabu@live.jp)
}

\section{Introduction}

In the previous paper [1], I define algorithmic manifolds simulating deterministic Turing machines and by determining the start point and end point of the algorithm in a $\mathrm{P}$ problem [2] on the algorithmic manifold, there is the optimal algorithm as the length minimizing geodesic between the start point and the end point, and the length minimizing geodesic can be derived by determining the start point and the end point also in a NP problem [2].

In this paper, I show that the possibility of translating algorithms from geodesics on algorithmic manifolds is equivalent to the halting problem of Turing machine [3]. I will also discuss the problems of translating from geodesics using existing algorithms.

2. Translatability problem of geodesics on algorithmic manifolds

It is assumed that an algorithm can be created by using some method from the geodesic on the algorithmic manifold, and the length $l$ of the algorithm and the data capacity $n$ which are a variable on the algorithmic manifold. An algorithm to determine whether or not this algorithm performs scheduled operation is necessary, but like the halting problem of Turing machine [3], it is an algorithm to be self-reference, so contradiction will occur. As a result, no such algorithm exists. Therefore, there is no algorithm that translates algorithms that perform predetermined actions from geodesics.

Conversely, by associating and combining existing countable algorithms that perform scheduled operation to the values of the length $l$ of the algorithm and the data capacity $n$ which are a variable of the algorithm manifold, an algorithm that performs scheduled operation may be created from the geodesic. However, in this case, since the length $l$ of the algorithm and the data capacity $n$ which are a real number are uncountable, it is impossible to correspond to existing algorithms that are countable. Therefore, this method cannot translate algorithms from geodesics.

\section{Conclusion}

From the geodesics on the algorithmic manifold, it was possible to show that there is no way to translate the algorithm in two ways. In these meanings, it can be said that it is difficult to translate to the polynomial-time algorithm that solves the existing NP 
problem even if the length minimizing geodesic exists when using the concept of algorithmic manifold.

\section{References}

[1] T. Yabu, "Algorithmic Manifold and Application to P versus NP Problem," IEICE Transactions on Information and Systems, in Posting, https://osf.io/43jcw/, 2016.

[2] S. Cook, “ The P versus NP Problem, " http://www.claymath.org/sites/default/files/pvsnp.pdf, 2000.

[3] A. Turing, “ On Computable Numbers, with an Application to the Entscheidungsproblem," Proceedings London Mathematical Society, Volume s2-42, Issue 1, 1937. 